\title{
White Dwarfs in Cataclysmic Variables: An Update
}

\author{
E. M. Sion ${ }^{1}$, P. Godon ${ }^{1}$ \\ ${ }^{1}$ Department of Astrophysics \& Space Science, Villanova University, 800 Lancaster Ave., Villanova, PA 19085, USA \\ Corresponding author: edward.sion@villanova.edu
}

\begin{abstract}
In this review, we summarize what is currently known about the surface temperatures of accreting white dwarfs in nonmagnetic and magnetic cataclysmic variables (CVs) based upon synthetic spectral analyses of far ultraviolet data. We focus only on white dwarf surface temperatures, since in the area of chemical abundances, rotation rates, WD masses and accretion rates, relatively little has changed since our last review, pending the results of a large HST GO program involving $48 \mathrm{CVs}$ of different CV types. The surface temperature of the white dwarf in SS Cygni is re-examined in the light of its revised distance. We also discuss new HST spectra of the recurrent nova $\mathrm{T}$ Pyxidis as it transitioned into quiescence following its April 2011 nova outburst.
\end{abstract}

Keywords: cataclysmic variables - dwarf novae - intermediate polars - spectroscopy - UV - individuals: T Pyx, CC Syg.

\section{Introductory Overview}

The white dwarfs in cataclysmic variables (hereafter CVWD) are the central engines of the observed outbursts, either as potential wells for the release of gravitational energy during accretion (dwarf nova - DN), or as the sites of explosive thermonuclear runaway (TNR) shell burning (classical novae), steady shell burning (supersoft X-ray binaries) or instantaneous collapse and total thermonuclear detonation if the WD reaches the Chandrasekhar limit (Type Ia supernova? SN Ia). Therefore, the accreting WDs serve as probes of explosive evolution and accretion physics and diffusion, as they bear the thermal, chemical and rotational imprint of their long term accretion and thermonuclear history (Sion 1991 \& 1995; Townsley \& Bildsten 2003; Townsley \& Gänsicke 2009).

Deeper physical insights however require a larger number of chemical abundances, rotation rates, surface temperatures, mass accretion rates, and masses for each spectroscopic subclass of CVs. Only then can any definitive conclusions be drawn. In order to adequately sample the parameter space $\left(M_{w d}, i, \dot{M}, T_{e f f}, P_{o r b}\right)$ of the DNs, Nova-like variables, and magnetic CVs, a large GO program was approved in Cycle 20 (B.Gänsicke, Principal Investigator) to secure high quality COS spectra for CV classes underrepresented in the current overall CV sample. The data is in hand and undergoing analysis at the time of this writing. Ultimately, we hope to obtain data for $>30 \mathrm{CVs}$ per class. Therefore, in this review our focus is restricted to white dwarf surface temperatures, since in the area of chemical abun- dances, rotation rates, WD masses and accretion rates, relatively little has changed since our last review in the 2011 Palermo meeting proceedings. In section 2, our FUV analysis techniques are briefly summarized, section 3 we address how the revised (shorter) distance to SS Cygni affects the results of our analysis of the FUSE + HST STIS spectra of SS Cygni by Sion et al. (2010), in section 4 we tabulate and display the current distribution of CVWD surface temperatures versus orbital period and in the final section, we include some remarks on new HST spectra of the recurrent nova T Pyxidis.

\section{Synthetic Spectral Analysis of FUV Spectra of CVWDs}

We have modeled the FUV spectra of WDs in CVs during DN quiescence and Nova-like low states from IUE, FUSE and HST (FOS, GHRS, STIS, COS) archival data, and through our ongoing collaboration with past HST surveys led by B.Gänsicke, and P. Szkody.

The IUE archival spectra and high quality FUSE and HST FUV data are fit with the latest versions of the TLUSTY/SYNSPEC model photosphere code and model accretion disk codes (Hubeny 1988; Hubeny \& Lanz 1995). We are taking into account the BL explicitly in our modeling of the FUV spectra of disk accreting systems, by replacing the very inner rings of the standard accretion disk model with high temperature rings in agreement with the temperature and density in the boundary layer. This improves the model fitting at the shorter wavelengths. As an example of our CVWD 
photosphere fitting, in Fig.1, we display a WD solar composition fit to the HST STIS spectrum of V442 Cen with $\mathrm{E}(\mathrm{B}-\mathrm{V})=0.10$. The WD model has $\mathrm{T}=47,000 \mathrm{~K}$ $\pm 2000 \mathrm{~K}$ and $\log (g)=8.3 \pm 0.2, V_{\sin (i)}=300 \mathrm{~km} / \mathrm{s} \pm 50$ $\mathrm{km} / \mathrm{s}$ (Sion et al. 2008).



Figure 1: The best-fitting single temperature WD fit to the HST/STIS spectrum of V442 Cen. The model consists of a $47,000 \mathrm{~K}$ WD with $\log (\mathrm{g})=8.3$ and scaled to a distance of $328 \mathrm{pc}$.

\section{The White Dwarf in SS Cygni:The VLBI and Corrected Hubble FGS Distance}

Schreiber \& Lasota (2007), and references therein, pointed out that the previously published Hubble FGS parallax of SS Cygni, with a distance of $166+/-12 \mathrm{pc}$, posed serious problems for the disc instability model (DIM) of dwarf nova outbursts because it would fail to explain the absolute magnitude during outburst. With the new VLBI-derived distance (Miller-Jones et al. 2013) to SS Cygni (114 pc), and the corrected HST distance (Nelan \& Bond 2013) of 120 pc instead of 166 pc, the concern for the validity of DIM is alleviated. An obvious question is: How does the new, shorter distance affect the analysis of Sion et al. (2010) regarding their detection of the WD during quiescence and their derivation of the WD's surface temperature from the modeling of FUSE + HST STIS spectra?

To answer this question, we have carried out model fits using the new distance in the range of $114 \mathrm{pc}$ to 120 pc. When we used single, steady state disk models, we obtained the same results as in Sion et al. (2010). The disk does not fit the flux in the shorter wavelengths unless the accretion rate is very large, which is inconsistent with dwarf nova quiescence, and the model-derived distance is far too large. When we combined a model disk with a model white dwarf photosphere (using the Bitner et al. WD mass as in Sion et al. (2010), the correct distance to SS Cygni was obtained for a low accretion rate and a lower temperature white dwarf. However, the fits with the WD + disk are inferior to the fits where the WD dominates the FUV flux. In order to match the best fit solution to the FUSE + HST STIS wavelength range in Sion et al. (2010), the white dwarf must have $T_{\text {eff }}=45000 \mathrm{~K}-50000 \mathrm{~K}$ for a distance of $112 \mathrm{pc}$ to 120 pc and a white dwarf mass $M_{w d}=0.95 M_{\odot}$. This value of the WD mass is within the error range of the Bitner et al. mass. Our conclusion about the $T_{\text {eff }}$ of the WD in SS Cygni during quiescence is essentially unchanged from Sion et al. (2010).

\section{The Current Distribution of CV White Dwarf Surface Temperatures Versus Orbital Period}

The effective temperatures $T_{\text {eff }}$ of the WDs (obtained during dwarf nova quiescence and nova-like low states when the WD is exposed), are the critical key to revealing the thermal response of the WD to mass accretion and the long term accretion rate $\langle\dot{M}>$ which, through compressional heating by the accreted material, is linked to the WD surface temperature (Sion 1995; Townsley \& Bildsten 2003; Townsley \& Gänsicke 2009).

In Table 1, we have tabulated what we regard as the most reliably secured WD surface temperatures. The first column gives the name of the CV, the second column the CV subtype, the third column, the orbital period in minutes, the fifth column the WD $T_{\text {eff }}$ and the sixth column, the reference for the temperature. These temperatures are derived from a variety FUV spectra and all are known with a precision of at least $3000 \mathrm{~K}$ and in the majority of cases, better that $+/-2000 \mathrm{~K}$. With the paucity of FUV-derived temperatures per CV subclass, one should not ignore usable FUV data from IUE, FUSE and HUT because the "quality" of the data is deemed inferior to HST STIS and COS.

The current distribution of WD $T_{\text {eff }}$ against the orbital period $P_{\text {orb }}$ is displayed in Fig.2. (see also Fig.4 in Townsley \& Gänsicke 2009). Note the continued relatively sparse coverage in temperature of the WDs in CVs above the period gap $\left(P_{\text {orb }}>3 h r\right)$, compared with the coverage below the period gap.

It is possible that the apparent trend toward higher temperatures with increasing $P_{\text {orb }}$ (i.e. higher long term average $\langle\dot{M}\rangle$ ) could be due to observational selection since the WD $T_{\text {eff }}$ 's were derived primarily in the FUV where the Planckian peak occurs for hotter accreting WDs. For example, Copperwheat et al. (2010) derive a $T_{\text {eff }}=10-15,000 \mathrm{~K}$ in the optical for the WD in the eclipsing DN IP Peg which has $P_{\text {orb }}=3.8 \mathrm{~h}$ while the eclipsing system SDSS1006 with $P_{\text {orb }}=4.46 \mathrm{~h}$, has $T_{\text {eff }}=16,500 \pm 2000 \mathrm{~K}$ (Southworth et al. 2009) in the optical. Both of these objects should contain much hotter WDs (higher $\langle\dot{M}>$ ) for their orbital periods. 
Table 1: The temperature of CV white dwarfs

\begin{tabular}{|c|c|c|c|c|}
\hline SYSTEM & CV TYPE & $P_{\text {orb }}(\mathrm{h})$ & $T_{e f f}(\mathrm{~K})$ & REF \\
\hline SDSS1507 & $\mathrm{DN} / \mathrm{SU}$ & 1.11 & 11000 & Szkody et al. (2010a) \\
\hline GW Lib & $\mathrm{DN} / \mathrm{WZ}$ & 1.280 & 14700 & Szkody et al. (2002a) \\
\hline BW Scl & DN? & 1.304 & 14800 & Gänsicke et al. (2005) \\
\hline LL And & $\mathrm{DN} / \mathrm{WZ}$ & 1.321 & 14300 & Howell et al. (2002) \\
\hline PQ And & $\mathrm{DN} / \mathrm{SU}$ & 1.34 & 12000 & Szkody et al. (2010a) \\
\hline EF Eri & $\mathrm{AM}$ & 1.350 & 9500 & Szkody et al. (2010b) \\
\hline SDSS J1610-0102 & DN? & 1.34 & 14500 & Szkody et al. (2007) \\
\hline V455 And & $\mathrm{DN} / \mathrm{SU}$ & 1.35 & 10500 & Szkody et al. (2013) \\
\hline HS2331+3905 & $\mathrm{DN}$ & 1.351 & 10500 & Araujob-Betancor et al. (2005a) \\
\hline AL Com & $\mathrm{DN} / \mathrm{WZ}$ & 1.361 & 16300 & Szkody et al. (2003) \\
\hline WZ Sge & $\mathrm{DN} / \mathrm{WZ}$ & 1.361 & 14900 & Sion et al. (1995) \\
\hline SW UMa & $\mathrm{DN} / \mathrm{SU}$ & 1.364 & 13900 & Gänsicke et al. (2005) \\
\hline SDSS0919 & $\mathrm{DN}$ & 1.36 & 13500 & Szkody (2014) \\
\hline SDSS1035 & DN? & 1.37 & 10500 & Southworth et al. (2006); Littlefair et al. (2006b) \\
\hline HV Vir & $\mathrm{DN} / \mathrm{WZ}$ & 1.370 & 13300 & Szkody et al. (2002b) \\
\hline SDSS1339 & $\mathrm{DN}$ & 1.38 & 12500 & Szkody et al. (2010a) \\
\hline SDSS2205 & $\mathrm{DN}$ & 1.38 & 15000 & Szkody et al. (2010a) \\
\hline WX Cet & $\mathrm{DN} / \mathrm{WZ}$ & 1.399 & 13500 & Sion et al. (2003) \\
\hline T Leo & $\mathrm{DN} / \mathrm{SU}$ & 1.41 & 16000 & Hamilton \& Sion (2004) \\
\hline EG Cnc & $\mathrm{DN} / \mathrm{WZ}$ & 1.44 & 12300 & Southworth et al. (2006) \\
\hline XZ Eri & $\mathrm{DN} / \mathrm{SU}$ & 1.468 & 15000 & Szkody et al. (2010a) \\
\hline SDSS1514 & $\mathrm{DN}$ & 1.48 & 10000 & Szkody (2014) \\
\hline DP Leo & $\mathrm{AM}$ & 1.497 & 13500 & Schwope et al. (2002) \\
\hline V347 Pav & $\mathrm{AM}$ & 1.501 & 11800 & Araujob-Betancor et al. (2005b) \\
\hline BC UMa & $\mathrm{DN} / \mathrm{SU}$ & 1.503 & 15200 & Gänsicke et al. (2005) \\
\hline EK $\operatorname{Tr} A$ & $\mathrm{DN} / \mathrm{SU}$ & 1.509 & 18000 & Godon et al. (2008) \\
\hline VY Aqr & $\mathrm{DN} / \mathrm{WZ}$ & 1.514 & 14500 & Sion et al. (2003) \\
\hline OY Car & $\mathrm{DN} / \mathrm{SU}$ & 1.515 & 15000 & Cheng et al. (2000) \\
\hline SDSS0131 & $\mathrm{DN} / \mathrm{SU}$ & 1.63 & 14500 & Szkody et al. (2010a) \\
\hline VV Pup & $\mathrm{AM}$ & 1.674 & 11900 & Araujob-Betancor et al. (2005b) \\
\hline V834 Cen & $\mathrm{AM}$ & 1.692 & 14300 & Araujob-Betancor et al. (2005b) \\
\hline HT Cas & $\mathrm{DN} / \mathrm{SU}$ & 1.768 & 14000 & Wood et al. (1992) \\
\hline VW Hyi & $\mathrm{DN} / \mathrm{SU}$ & 1.783 & 22000 & Godon et al. (2008) \\
\hline CU Vel & $\mathrm{DN} / \mathrm{SU}$ & 1.88 & 18500 & Gänsicke \& Koester (1999) \\
\hline MR Ser & $\mathrm{AM}$ & 1.891 & 14200 & Araujob-Betancor et al. (2005b) \\
\hline BL Hyi & $\mathrm{AM}$ & 1.894 & 13300 & Araujob-Betancor et al. (2005b) \\
\hline ST LMi & $\mathrm{AM}$ & 1.898 & 10800 & Araujob-Betancor et al. (2005b) \\
\hline AR UMa & $\mathrm{AM}$ & 1.93 & 20000 & Schmidt et al. (2005) \\
\hline REJ1225 & $\mathrm{DN} / \mathrm{SU}$ & 1.99 & 12000 & Szkody et al. (2010a) \\
\hline EF Peg & $\mathrm{DN} / \mathrm{WZ}$ & 2.01 & 16600 & Howell et al. (2002) \\
\hline DV UMa & $\mathrm{DN} / \mathrm{SU}$ & 2.138 & 20000 & Feline et al. (2004) \\
\hline HU Aqr & $\mathrm{AM}$ & 2.084 & 14000 & Gänsicke (1999) \\
\hline QS Tel & $\mathrm{AM}$ & 2.332 & 17500 & Rosen et al. (2001) \\
\hline SDSS J1702+3229 & $\mathrm{DN} / \mathrm{SU}$ & 2.402 & 17000 & Littlefair et al. (2006a) \\
\hline TU Men & $\mathrm{DN}$ & 2.813 & 28000 & Sion et al. (2008) \\
\hline AM Her & $\mathrm{AM}$ & 3.094 & 19800 & Gänsicke et al. (1995) \\
\hline MV Lyr & $\mathrm{NL} / \mathrm{VY}$ & 3.176 & 45000 & Godon et al. (2012) \\
\hline DW UMa & $\mathrm{NL} / \mathrm{VY}$ & 3.279 & 50000 & Araujob-Betancor et al. (2003) \\
\hline TT Ari & $\mathrm{NL} / \mathrm{VY}$ & 3.301 & 39000 & Gänsicke et al. (1999) \\
\hline IP Peg & $\mathrm{DN}$ & 3.80 & 15000 & Southworth et al. (2009) \\
\hline VY Scl & NL & 3.99 & 45000 & Hamilton \& Sion (2008) \\
\hline V1043 Cen & $\mathrm{AM}$ & 4.190 & 15000 & Araujob-Betancor et al. (2005a) \\
\hline WW Cet & $\mathrm{DN}$ & 4.220 & 26000 & Godon et al. (2008) \\
\hline UGem & $\mathrm{DN} / \mathrm{UG}$ & 4.246 & 30000 & Sion et al. (2001) \\
\hline SSAur & $\mathrm{DN} / \mathrm{UG}$ & 4.391 & 34000 & Godon et al. (2012) \\
\hline SDSS1006 & $\mathrm{DN}$ & 4.46 & 16500 & Southworth et al. (2009) \\
\hline V895 Cen & $\mathrm{AM}$ & 4.765 & 14000 & Araujob-Betancor et al. (2005b) \\
\hline RX And & $\mathrm{DN} / \mathrm{ZC}$ & 5.037 & 34000 & Sion et al. (2001) \\
\hline SS Cyg & $\mathrm{DN} / \mathrm{UG}$ & 6.60 & 47000 & Sion et al. (2010) \\
\hline VY Scl & $\mathrm{NL}$ & 3.99 & 45000 & Hamilton \& Sion (2008) \\
\hline EM Cyg & $\mathrm{DN} / \mathrm{ZC}$ & 6.98 & 40000 & Godon et al. (2012) \\
\hline TT Crt & $\mathrm{DN}$ & 7.30 & 29000 & Sion et al. (2008) \\
\hline RU Peg & $\mathrm{DN}$ & 8.99 & 70000 & Godon et al. (2012) \\
\hline V442 Cen & $\mathrm{DN}$ & 11.04 & 47000 & Sion et al. (2008) \\
\hline
\end{tabular}




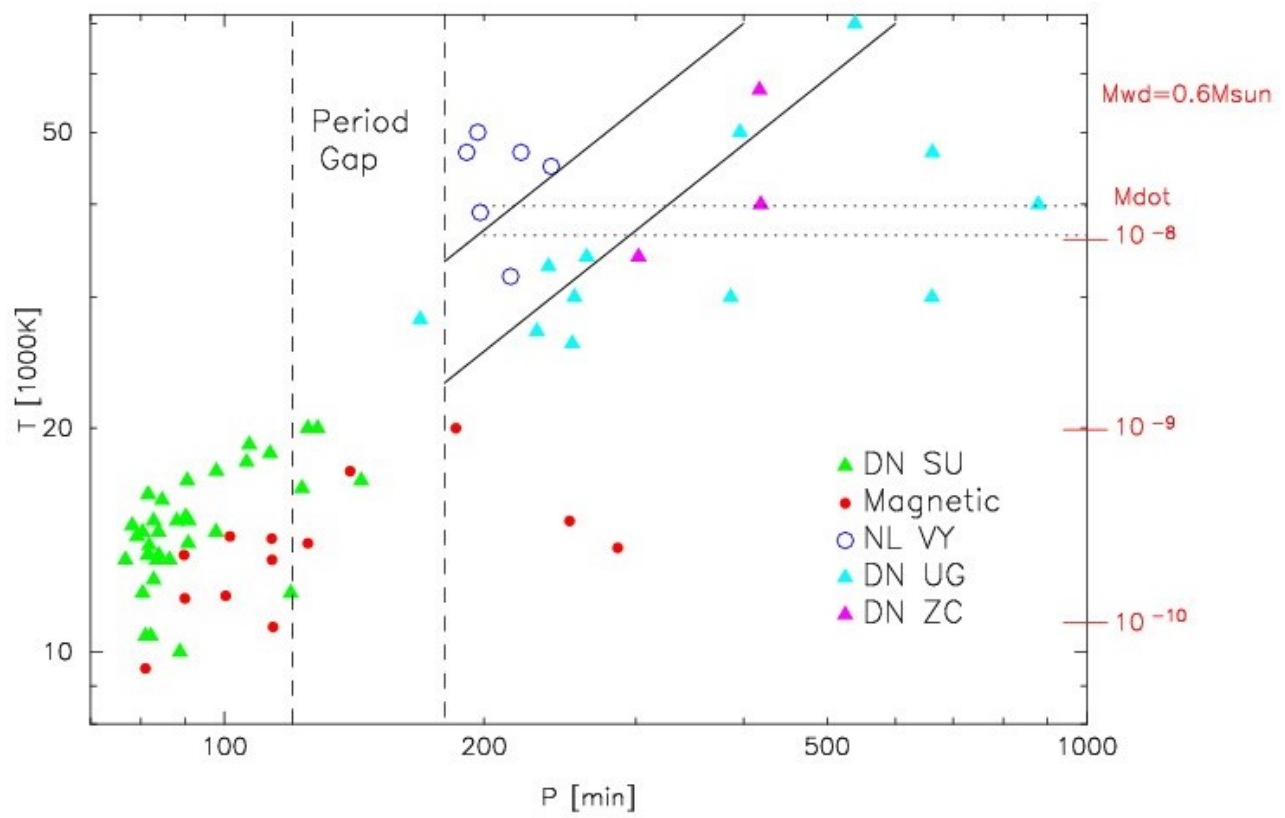

Figure 2: Effective White Dwarf Temperature as a function of the orbital period. The references for the indivdual temperatures can be found in Sion et al. 2008, Townsley \& Gänsicke 2009, Araujo-Betancor 2005a $\& \mathrm{~b}$ and references therein). The traditional magnetic braking above the period gap is shown between the parallel diagonal solid lines. On the right hand side are the time-averaged accretion rates correpsonding to the temperature scale on the left hand side of the diagram. Shown for comparison between the dotted lines is the long term evolutionary path of a 0.8 solar mass white dwarf (with an initial core temperature of 30 million degrees $\mathrm{K})$ which has undergone 1000 nova outburst cycles accreting at the long term rate of $10^{-8} M_{\odot} / \mathrm{yr}$

\section{The HST COS + STIS Spectra of the Recurrent Nova T Pyxidis:A Progress Report}

The interstellar reddening $\mathrm{E}(\mathrm{B}-\mathrm{V})$ of the recurrent nova $\mathrm{T}$ Pyxidis is a critical parameter in the determination of the best-fitting model parameters.

The UV spectra exhibit a minimum near $2175 \mathrm{~A}$ which is due to the interstellar extinction. Since the GALEX spectrum is the most reliable (i.e. highest $\mathrm{S} / \mathrm{N}$ ratio) in that wavelength region, we used the GALEX spectrum to determine $\mathrm{E}(\mathrm{B}-\mathrm{V})$. The value of $\mathrm{E}(\mathrm{B}-\mathrm{V})$ for which the $2175 \mathrm{~A}$ feature disappears from the dereddened spectrum, $\mathrm{E}(\mathrm{B}-\mathrm{V})=0.35$, is taken as the $\mathrm{E}(\mathrm{B}-\mathrm{V})$ value towards T Pyx (see Fig.3). We use this value to deredden the IUE, GALEX and HST spectra, and we also consider the effects of different reddening values on our results.

The HST STIS and COS spectra obtained in December 2012 and July 2013 are identical. Thus, we co-added them to improve the signal-to-noise $(\mathrm{S} / \mathrm{N})$. We have found that the pre-outburst IUE and GALEX spectra together with the HST post-outburst spectrum. We note that the UV flux has remained constant not only before the outburst, but it has now come back precisely to the same value. This is an indication that

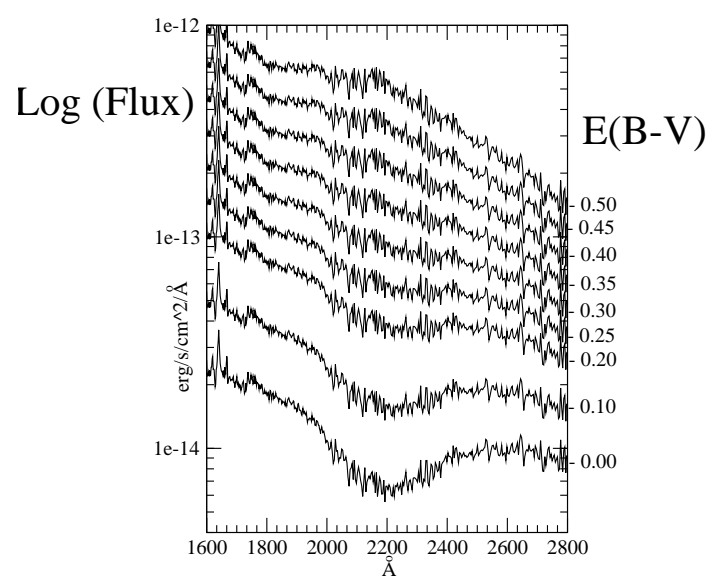

Figure 3: The merged Galex-IUE spectrum of T Pyx has been dereddened for different values of $\mathrm{E}(\mathrm{B}-\mathrm{V})$ as indicated on the right. The $2175 \AA$ feature associated with the reddening is clearly seen in absoprtion for low balues of $\mathrm{E}(\mathrm{B}-\mathrm{V})$, and it appears as extra flux for large values of $\mathrm{E}(\mathrm{B}-\mathrm{V})$. We deduce that the reddening towards T Pyx must be $\mathrm{E}(\mathrm{B}-\mathrm{V})=0.35$, the value for which the $2175 \AA$ feature vanishes. 
White Dwarfs in Cataclysmic Variables: An Update

the mass accretion rate remained constant before and after the outburst (see Godon et al. 2014).

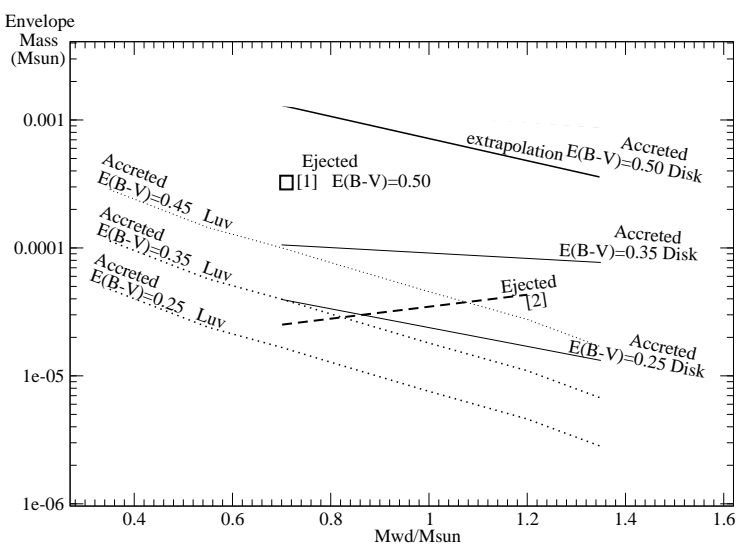

Figure 4: The mass of the accreted envelope and ejected envelope are shown as a function of the WD mass for different values of the reddening for T Pyx. Our disk model results are drawn with a solid line ("Disk"). The lower limit of the accreted envelope inferred from the UV flux is shown (dotted line, "Luv"). In comparison we show the ejected envelope [1] as estimated by Nelson et al. (2012) (square symbol), as well as [2] computed in Patterson et al. (2013) (dashed line). The accreted envelope is larger than the ejected envelope, except for a value of $\mathrm{E}(\mathrm{B}-\mathrm{V})=0.25$ combined with a WD mass $\sim 0.9 M_{\odot}$ and larger. However, the best results were obtained for a larger value of the reddening $E(B-V)>0.30$.

Using the value of the reddening we derived, together with the new distance estimate of $4.8 \mathrm{kpc}$ (Sokoloski et al. 2013), we fit the observed (back-toquiescence) HST spectra with disk models for different WD masses. We then computed the accreted mass over a period of $44 \mathrm{yrs}$, which we then compared to the estimates of the ejected envelope mass (during the 2011 outburst). We recapitulate our results in Fig.4, where we also consider different reddening values for the sake of completness. Our main finding is that the mass of the accreted material between the last two outbursts is larger than the mass of the ejected envelope in the last outbursts, unless the reddening is $\mathrm{E}(\mathrm{B}-\mathrm{V})=0.25$ (or smaller) $A N D$ the mass of the $\mathrm{WD}$ is $\sim 0.9 M_{\odot}$ or larger. However, such a small value of the reddening leads to a very bad fit and has therefore to be rejected.

\section{Acknowledgement}

This research is supported by HST grants GO12799.01A and GO- 12890.01A, both to Villanova Uni- versity. The Archival data (IUE, FUSE \& Galex) analysis was supported by the National Aeronautics and Space Administration (NASA) under grant number NNX13AF11G issued through the Office of Astrophysics Data Analysis Program (ADAP) to Villanova Univesity.

\section{References}

[1] Araujo-Betancor, S., et al.: 2005a, A\&A, 430, 629

[2] Araujo-Betancor, S., et al.: 2005b, ApJ, 622, 589

[3] Araujo-Betancor, S., et al.: 2003, ApJ, 583, 437 doi:10.1086/345098

[4] Cheng, F. H., et al.: 2000, ApJ, 542, 1064

[5] Copperwheat, C., Marsh, T. R., Dhillon, V., Littlefair, S.. Hickman, R., Gnsicke, B., Southworth, J.: 2010, MNRAS, 402, 1824

[6] Feline, W. J., et al.: 2004, MNRAS, 347, 1173

[7] Gänsicke, B. T., et al.: 2005, ApJ, 629, 451 doi:10.1086/431271

[8] Gänsicke, B. T.: 1999, in Annapolis Workshop on Magnetic Cataclysmic Variables, ed. C. Hellier \& K. Mukai (ASP Conf. Ser. 157), 261, 272

[9] Gänsicke, B. T., Beuermann, K., \& de Martino, D.: 1995, A\&A, 303, 127

[10] Gänsicke, B. T. \& Koester, D.: 1999, A\&A, 346, 151

[11] Gänsicke, B. T., et al.: 1999, A\&A, 347, 178

[12] Godon, P., et al.: 2004, ApJ, 602, 336

[13] Godon, P., et al.: 2008, Apj, 679, 1447 doi:10.1086/587504

[14] Godon, P., et al.: 2012, Apjs, 203, 29 doi:10.1088/0067-0049/203/2/29

[15] Godon, P., et al.: 2014, ApJL, in press (arXiv:1402.0128)

[16] Hamilton, R.,\& Sion, E.: 2004, PASP, 116, 926

[17] Hamilton, R.,\& Sion, E.: 2008, PASP, 120, 165 doi:10.1086/528939

[18] Howell, S. B., et al.: 2002, ApJ, 575, 419 doi:10.1086/341213

[19] Hubeny, I.: 1988, Comput. Phys. Comm., 52, 103

[20] Hubeny, I., \& Lanz, T.: 1995, Apj, 439, 875

[21] Littlefair, S. P., et al.: 2006a, MNRAS, 371, 1435

[22] Littlefair, S. P., et al.: 2006b, Science, 314, 1578 
[23] Miller-Jones, J. C. A., et al.: 2013, Science, 340, 950

[24] Nelan, E., \& Bond, H.E.: 2013, ApJL, 773, L26

[25] Nelson, T. et al. 2012,: Apj, submitted, [astro-Ph arXiv:1211.3112]

[26] Patterson, J. et al.: 2013, ASP Conf. Ser. Stellar Novae: Past and Future Decades, ed. P.A. Woudt, \& V.A.R.M. Ribeiro, in press (7pp)

[27] Rosen, S. R., et al.: 2001, MNRAS, 322, 631 doi:10.1046/j.1365-8711.2001.04141.x

[28] Schmidt, G. D., et al.: 2005, ApJ, 630, 1037 doi:10.1086/431969

[29] Schreiber, M.R., \& Lasota, J.-P.: 2007, A\&A, 473,897

[30] Schwope, A. D., et al.: 2002, A\&A, 392, 541

[31] Sion, E.M.: 1991, AJ, 102, 295

[32] Sion, E.M.: 1995, ApJ, 438, 876

[33] Sion, E.M.: 1999, PASP, 111, 532

[34] Sion, E.M. et al.: 1995, ApJ, 439, 957

[35] Sion, E.M., et al.: 2008, Apj, 681, 543 doi:10.1086/586699

[36] Sion, E. M., Cheng, F., Huang, M., Hubeny, I., \& Szkody, P.: 1996, ApJ, 471, L41

[37] Sion, E. M., et al.: 2003, ApJ, 583, 907

[38] Sion, E. M., Szkody, P., Gänsicke, B., Cheng, F. H., LaDous, C., \& Hassall, B.: 2001, ApJ, 555, 834
[39] Sion, E.M., Godon, P., Myszka, J., \& Blair, W.P.: 2010, ApJL, 716, L157 doi:10. 1088/2041-8205/716/2/L157

[40] Sokoloski, J., et al.: 2013, ApJ, 770, L33 doi:10.1088/2041-8205/770/2/L33

[41] Southworth, J., et al.: 2006, MNRAS, 373, 687 doi:10.1111/j.1365-2966.2006.11042.x

[42] Southworth, J. et al.: 2009, A\&A, 507, 929

[43] Szkody, P., Gänsicke, B. T., Howell, S. B., \& Sion, E. M.: 2002a, ApJ, 575, L79

[44] Szkody, P., Gänsicke, B. T., Sion, E. M., \& Howell, S. B.: 2002b, ApJ, 574, 950

[45] Szkody, P.et al. 2007: ApJ, 658, 1188 doi:10.1086/511854

[46] Szkody, P., et al.: 2010a, ApJ, 710, 64

[47] Szkody, P., et al.: 2010b, ApJ, 716, 1531 doi:10.1088/0004-637X/716/2/1531

[48] Szkody, P., et al.: 2013, ApJ, 775, 66 doi:10.1088/0004-637X/775/1/66

[49] Szkody, P.: 2014, private communication

[50] Townsley, D.,\& Bildsten, L.: 2003, Apj, 596, L227

[51] Townsley, D., \& Gänsicke, B.: 2009, Apj, 693, 1007 doi:10.1088/0004-637X/693/1/1007

[52] Wood, J. H., Horne, K., \& Vennes, S.: 1992, ApJ, 385,294 